DOE/ER/13479--2003F

\title{
FINAL TECHNICAL REPORT
}

\author{
DOE Grant Number \\ DE-FG03-86ER13479-A014
}

Stanford Account Number

2FBW402

\author{
Grant Period \\ 5/1/99 to $4 / 30 / 03$
}

Principle Investigator

John Ross 


\title{
DOE Grant Number: DE-PG03-86ER13479-A014
}

\author{
Stanford Account Number: 2FBW402
}

Grant Period: $51 / 99$ to $4 / 30 / 03$

\author{
Principle Investigator: John Ross
}

Extensive progress was made in the period covered by this Technical Report in the areas of chemical kinetics of non-linear systems; spatial structures, reaction - diffusion systems, and Thermodynamic and stochastic theory of electrochemical and general systems. The work is summarized by subjects of published articles:

\section{Linear Free Energy Relations and Reversible Stretched Exponential Kinetics in Systems with Static or Dynamical Disorder}

Stretched exponential relaxation is the result of the existence of a large number of relaxation channels, any of them having a very small probability of being open. It is shown that the stretched exponential kinetics obeys a type of linear free energy relation. The configuration entropy generated by the random distribution of channels is a linear function of the activation energy of the channel with the slowest relaxation rate and highest energy barrier. This property of stretched exponential relazation is used for studying the multichannel first-order relaxation kinetics of reversible processes. By combination of the linear free energy relationship with the principle of detailed balance, a generalized kinetic law of the stretched exponential type is derived, which provides a theoretical justification for its prior use in the literature for fitting experimental data. The theory is extended to reversible processes with dynamical disorder. In this case there is no simple analogue of the free energy relationship suggested for systems with static disorder; however, stretched exponencial kinetics can be investigated by using a stochastic Liouville equation. It is shown that for a process with dynamical disorder it is possible that in the long time limit the system evolves toward a nonequilibrium frozen state rather than toward thermodynamic equilibrium. We also study the concentration fluctuations for reversible chemical processes in systems with static or dynamical disorder. A set of fluctuation-dissipation relations is derived for the factorial moments of the number of molecules, and it is shown that for both typos of disorder the composition fluctuations are intermittent. For the global characterization of the average kinetic behavior of reversible processes occurring in disordered systems we introduce an average lifetime distribution of the transient regime and an effective rate coefficient. The analytic properties of these two functions are investigated for systems with both static and dynamical disorder. Finally, the theory is extended to the case of one-channel thermally activated processes with random energy barriers. We emphasize that our theoretical approach, unlike other theories of stretched exponential relaxation, does not make use of the steepest descent approximation for computing the average kinetic curves: our results are exact in a limit of the thermodynamic type, for which the total number of relaxation channels tends to infinity and the probability that a relaxation channel is open tends to zero, with the constraint that the average number of open channels is kept constant. This research was published in Journal of Physical Chemistry (368) 


\section{Nonlinear Kinetics and New Approaches to Complex Reaction Mechanisms}

This paper reviews recent developments in the field of nonlinear chemical kinetics. Five topics are dealt with: (a) new approaches to complex reaction mechanisms, stoichiometric network analysis, classification of chemical oscillators and formulation of their mechanisms by deduction from experiments, and correlation metric construction of reaction pathways from measurements; (b) thermodynamic and stochastic theory of nonequilibrium processes, the eikonal approximation, the evaluation of stochastic potentials, experimental tests of the thermodynamic and stochastic theory of relative stability, and fluctuation-dissipation relations in nonequilibrium chemical systems; $(c)$ chemical kinetics and cellular aucomata and lattice gas automata; $(d)$ theoretical approaches and experimental studies of stochastic resonance in chemical kinetics; and (e) rate processes in disordered systems, stochastic Liouville equations, stretched exponential relaxation in disordered systems, and universality classes for rate processes in systems with static or dynamic disorder. This project was published in Annual Reviews of Physical Chemistry (369)

\section{Spatial Correlations near Turing Instabilities: Criteria for Wavenumber Selection}

We analyze the probability distribution and spatial correlations around a stationary state of a general reaction-diffusion system. The stochastic description is based on a multivariate master equation. We use a WKB expansion of the probability density and determine the leading term, the stochastic potential, to second order in deviations from a homogeneous stationary state. For a system below, but near, a Turing instability, the spatial correlations become long range and display a macroscopic structure that will emerge after the bifurcation. We derive explicit expressions for both the probability density and the correlation function. For systems close to the instability, the correlation function is approximately an exponentially damped cosine function. We derive explicit expression for the correlation length and the amplitude of the correlation function; they are inversely proportional to the square root of the largest eigenvalue of the deterministic system. Our approach differs from earlier work in that systems with many chemical species are treated, asymptotic approximations are derived, correlations are given a geometrical picture in terms of eigenvectors of the Jacobian of an associated Hamiltonian system, and higher order terms in the stochastic potential are possible to obtain analytically (which we do not pursue). Results of the theoretical analysis are applied to the Sel'kov model. Exact and approximate solutions at lowest order agree well. This research was published in Journal of Physical Chemistry (370)

\section{Measurements of Phase Response in an Oscillatory Reaction and Deduction of Components of the Adjoint Eigenvector}

We present the first experiments that use the phase response method to determine components of the adjoint eigenvector (of the Jacobian matrix of the linearized system) of an oscillating reaction system. The Briggs-Rauscher reaction was studied near a supercritical Hopf bifurcation. Phase response curves for $\mathrm{I}^{-}$and $\mathrm{Mn}^{2+}$ have been determined, and from them corresponding components of the adjoint eigenvector have been deduced. The relative magnitudes and difference in arguments of these components agree reasonably well with those from a reduced model of the Briggs-Rauscher reaction, whereas agreement with results from quenching experiments is mixed. This project was published in Joumal of Physical Chemistry (374) 


\section{Statistical Ensemble Approach and Fluctuation-Dissipation Relations for Multivariable Chemical Systems Far from Equilibrium}

A statistical ensemble approach and an ensemble master equation are incroduced for the study of concentration fluctuations in multivariable chemical systems far from equilibrium. The theory describes the stochastic properties of the numbers of replicas of the system characterized by different compositions. We give a general analytic solution of the ensemble master equation and investigate the relationships between the ensemble master equation and the one-system master equation. A condition of mesoscopic time reversal (mesoscopic reversibility) is introduced for a reference system; mesoscopic reversibility is less restrictive than microscopic reversibility. For systems with mesoscopic time reversal the general theory turns into a simple form and, in the thermodynamic limit, we derive an exact expression for the stochastic potential attached to the one system master equation. We study the stochastic properties of the numbers of the reaction events both for system with or without mesoscopic time reversal. The condition of mesoscopic time reversal can be described by an extremum condition: if the contributions of different reactions to the total number of reaction events are constant, then the dispersions of the net numbers of the reaction events have minimum values for mesoscopic reversibility. A set of fluctuation-dissipation relations is derived for multivariable chemical systems, based on the use of the reaction extents as state variables of the system. We also consider systems that do not obey the condition of mesoscopic time reversal and measure the departure of a chemical process from mesoscopic reversibility in terms of a set a functions, which are proportional to the average values of the net numbers of the reaction events. In terms of these functions we derive a set of fluctuation-dissipation relations that establish a general relationship among the rates and the reaction affinities of the different reactions occurring in the system. A component of the dissipation function of the process is computed by using these fluctuation-dissipation relations. This research was published in the Joumal of Physical Chemistry (375)

\section{Master Equation Approach to Synchronization in Diffusion-Coupled Nonlinear Oscillators}

We study the influence of internal fluctuations on phase synchronization in oscillatory reactiondiffusion systems through a master equation approach. In the limit of large system size, the probability density is analyzed by means of the eikonal approximation. This approximation yields a Hamilton-Jacobi equation for the stochastic potential, which may be reduced to coupled nonlinear diffusion equations for the phase of oscillation and (conjugate) "momentum." We give explicit expressions for the coefficients of these equations in terms of averages over the deterministic periodic orbit. For one-dimensional systems, we obtain an explicit solution for the stationary stochastic potential: the width in phase, which is defined as the root mean square fluctuation in phase, characterizes the roughness of phase locking, and diverges with the system size $L$ according to a power law $w-L^{\alpha}$, with $\alpha=1 / 2$. To study higher-dimensional systems, we show that the eikonal approximations of the diffusion-coupled oscillator problem and the KardarParisi-Zhang (KPZ) equation (in the limit of small noise intensity) are equivalent. The KPZ equation governs some forms of surface growth, and the height of a growing front corresponds to the phase (the $2 \pi$ periodicity in phase is ignored) in the diffusion-coupled oscillator problem. From the equivalence, we obtain the result that spatially synchronized states may exist only in systems with a spatial dimension greater than or equal to 3; for dimensions 1 and 2, a "rough" state exists in which the width (in phase) diverges algebraically with the system size, $\alpha>0$. This project was published in Physical Review E (379) 


\section{Statistical fractal adsorption isotherms, linear energy relations, and power-law trapping-time distributions in porous media}

Drazer and Zanette (Phys. Rev. E 60, 5858, 1999) have reported on interesting experiments which show that trapping-time discributions in porous media obey a scaling law of the negative power-law type. Unfortunately, their theoretical interpretation of the experimental data has physical and mathematical inconsistencies and errors. Drazer and Zanette assume the existence of a distribution of local adsorption isotherms for which the random parameter is not a thermodynamic function, but a kinetic parameter, the trapping time. Moreover, they mistakenly identify the reciprocal value of a rate coefficient with the instantaneous (fluctuating) value of the trapping time. Their approach leads to mathematically inconsistent probability densities for the crapping times, which they find to be non-normalizable. We suggest a different theory, which is physically and mathematically consistent. We start with the classical pacch approximacion, which assumes the existence of a distribution of adsorption heats, and introduce two linear energy relationships between the activation energies of the adsorption and desorption processes and the adsorption heat. If the distribution of the adsorption heat obeys the exponential law of Zeldovich and Roghinsky, then both the adsorption isotherm and the probability density of trapping times can bo evaluated analytically in terms of the incomplete beta and gamma functions, respoctively. Our probability density of the crapping times is mathematically consistent; that is, it is nonnegative and normalized to unity. For large times it has a long tail, which obeys a scaling law of the negative power-law type, which is consistent with the experimental data of Drazer and Zanette. By using their data we can evaluate the numerical values of the proportionality coefficients in the linear energy relations. The theory suggests that experimental study of the temperature dependence of the fractal exponents helps to elucidate the mechanism of the adsorption-desorption process. This research was published in Physical Review E (380)

\section{Levy diffusion in a force field, Huber relaxation kinetics, and nonequilibrium thermodynamics: $H$ theorem for enhanced diffusion with Levy white noise}

A characteristic functional approach is suggested for Levy diffusion in disordered systems with extemal force fields. We study the overdamped motion of an ensemble of independent particles and assume that the force acting upon one particle is made up of two additive components: a linear term genetated by a harmonic potential and a second term generated by the interaction with the disordered system. The stochastic properties of the second term are evaluated by using Huber's approach to complex relaxation (Phys. Rev. B 31, 6070;1985). We assume that the interaction between a moving particle and the environment can be expressed by the contribution of a large number of relaxation channels, each channel having a very small probability of being open and obeying Poisson statistics. Two types of processes are investigated: (a) Levy diffusion with static disorder for which the fluctuations of the random force are frozen and last forever and (b) diffusion with strong dynamic disorder and independent Levy fluctuations (Levy white noise). In both cases we show that the probability distribution of the position of a diffusing particle tends towards a stationary nonequilibrium form. The characteristic functional of concentration fluctuations is evaluated in both cases by using the theory of random point processes. For large times the fluctuations of the concentration field are stationary and the corresponding probability density functional can be evaluated analytically. In this limit the fluctuations depend on the distribution of the total number of particles but are independent of the initial positions of the particles. We show that the logarithm of the stationary probability functional plays the role of a nonequilibrium thermodynamic potential, which has a structure similar to the Helmholtz free energy in equilibrium thermodynamics: it is made up of the sum of an energetic component, depending on the external mechanical potential, and of an entropic 
component, depending on the concentration field. We show that the conditions for the existence and stability of the nonequilibrium steady state, which emerges for large times, can be expressed in terms of the stochastic potential. For Levy white noise the average concentration field can be expressed as the solution of a fractional Fokker-Planck equation. We show that the stochastic potential is a Lyapunov function of the fractional Fokker-Planck equation, which ensures that all transient solutions for the average concentration field tend towards a unique stationary form. Rhis research project was published in Physical Review (381)

\section{New Approaches to Complex Reaction Mechanisms}

Complex reaction mechanisms, as for example the citric acid cycle, consist of many elementary reaction steps; each step can be described by listing the reactants and products in that step, taken to occur in a single collision event. Further each step may have catalysts as well as positive and negative effectors on these catalysts. The traditional approach to investigating complex reaction mechanisms consists of identifying as many participating species as possible (reactants, products, intermediates), isolating each elementary reaction step, and determining the stoichiometry and kineties of each step. From these measurements and any others, such as isotope studies, reaction mechanisms are guessed by intuition, prior experience, analogies, etc. and the predictions of the hypothesized mechanism are checked with experiments. There exists no agreed-upon prescription for this approach, and hence it is not surprising that for many mechanisms discussions persist for years. In this article we study several new approaches to the establishment of complex reaction mechanisms presented in the last few years. The goal of these approaches is the formation of methods for deducing reaction mechanisms, or perhaps only reaction pathways that show the connections among species due to reactions, from prescribed measurements. This study was published in 'Design Principles for the Immune System and Other Distributed Autonomous Systems, Lee Segel and Irun Cohen, eds, Oxford University Press, Oxford, 2000 (382)

\section{On the deduction of chemical reaction pathways from measurements of time series of concentrations}

We discuss the deduction of reaction pathways in complex chemical systems from measurements of time series of chemical concentrations of reacting species. First we review a technique called correlation metric construction (CMC) and show the construction of a roaction pathway from measurements on a part of glycolysis. Then we present two new improved methods for the analysis of time series of concentrations, entropy metric construction (EMC), and entropy reduction method (ERM), and illustrate (EMC) with calculations on a model reaction system. This Research was published in Chaos (384)

\section{Effective Medium Approximation of Taylor Transport in Systems with Static Disorder}

The article presents studies on the Taylor transport in disordered systems. We assume that a moving particle can exist in different states and that for each state its transport properties are described by a different linear evolution operator. The transition from one state to another is described by a Markov process in continuous time. The transition rates of the Markov process depend on a set of variable parameters that are randomly selected from a known probability density. This type of transport model is of incerest in connection with the study of multiphasic transport, for example, in the case of chromatographic separation, neutron migration in nuclear reactions, or neutrino flow in astrophysical problems. We consider two different types of effective medium approximations: (1) a global approach, which consists of solving the Taylor 
problem for a given set of values of the of the random parameters, followed by averaging the result, and (2) a local approach, for which the averaging is done for small macroscopic regions of the system, which leads to a system of agedependent master equations. We show that the averaging in the global approach induces an arcificial collective behavior that results in ballistic diffusion. The local approach may lead either to normal or dispersive transport. We apply our theory to the experiment of Drazer and Zanette (Phys. Rev. E. 1999, 60, 5858) and show that that the local approach is in agreement with experimental data. This research was published in the Joumal of Physical Chemistry (388)

\section{Bayesian analysis of systems with random chemical composition: Renormalization-group approach to Dirichlet distributions and the statistical theory of dilution}

We investigate the statistical properties of systems with random chemical composition and try to obtain a theoretical derivation of the self-similar Dirichlet distribution, which is used empirically in molecular biology, environmental chemistry, and geochemistry. We consider a system made up of many chemical species and assume that the statistical distribution of the abundance of each chemical species in the system is the result of a succession of a variable number of random dilution events, which can be described by using the renormalization-group theory. A Bayesian approach is used for evaluating the probability density of the chemical composition of the system in terms of the probability densities of the abundances of the different chemical species. We show that for large cascades of dilution events, the probability density of the composition vector of the system is given by a self-similar probability density of the Dirichlet type. We also give an altemative formal derivation for the Dirichlet law based on the maximum entropy approach, by assuming that the average values of the chemical potentials of different species, expressed in terms of molar fractions, are constant. Although the maximum entropy approach leads formally to the Dirichlet distribution, it does not clarify the physical origin of the Dirichlet statistics and has serious limitations. The random theory of dilution provides a physical picture for the emergence of Dirichlet statistics and makes it possible to investigate its validity range. We discuss the implications of our theory in molecular biology, geochemistry, and environmental science. This research project was published in Physical Review (389)

\section{Determination of Thermodynamic and Stochastic Potentials in Nonequilibrium Systems from Macroscopic Measurements}

For systems far from equilibrium, a potential determines the fluctuations from stable attractors, such as stationary states, and the thermodynamic approach to such states (Lyapunov function). For a reaction system with ionic reactions in a stationary state far from equilibrium, the imposition of a current displaces the system from that state. For such systems, we show that an electrochemical potential due to the imposed current, consisting of both a Nernstian term and a non-Nernstian term, directly yields the stochastic potential around its maximum to a good approximation, without knowledge of the reaction mechanism of the system. The approximation is good to the order of the percent difference between the Nernstian and non-Nernstian terms and can be used for evaluating the stochastic potential. For general systems, the imposition of a flow of intermediates also displaces the system from its stationary state to a new stationary state, which is a nonstationary state of the system without flow. The concentrations of the chemical species can be measured without time restraints, and by repeating various such displacements, sufficient data can be obtained to determine rate coefficients for a solution of a stationary master equation and its stochastic potential for a known or assumed reaction mechanism. Several experiments are 
suggested to test the consistency of the results with predictions of the master equation and, thus, to test that equation itself. This research was published in Journal of Physical Chemistry (397)

\section{Systematic derivation of reaction-diffusion equations with distributed delays and relations to fractional reaction-diffusion equations and hyperbolic transport equations: Application to the theory of Neolithic transition}

We introduce a general method for the systematic derivation of nonlinear reaction-diffusion equations with distributed delays. We study the interactions among different types of moving individuals atoms, molecules, quasiparticles, biological organisms, etc. The motion of each species is described by the continuous time random walk theory, analyzed in the literature for transport problems, whereas the interactions among the species are described by a set of transformation rates, which are nonlinear functions of the local concentrations of the different types of individuals. We use the time interval between two jumps, the transition time, as an additional state variable and obtain a set of evolution equations, which are local in time. In order to make a connection with the transport models used in the literature, we make transformations which eliminate the transition time and derive a set of nonlocal equations which are nonlinear generalizations of the so-called generalized master equations. The method leads under different specified conditions to various types of nonlocal transport equations including a nonlinear generalization of fractional diffusion equations, hyperbolic reaction-diffusion equations, and delay-differential reaction-diffusion equations. Thus in the analysis of a given problem we can fit to the data the type of reaction-diffusion equation and the corresponding physical and kinetic parameters. The method is illustrated, as a test case, by the study of the neolithic transition. We introduce a set of assumptions which makes it possible to describe the transition from hunting and gathering to agriculture economics by a differential delay reaction-diffusion equation for the population density. We derive a delay evolution equation for the race of advance of agriculure, which illustrates an application of our analysis. This research project was published in Physical Review (399)

\section{Electrochemical potentials in nonequlibrium steady states and oscillatory systems: General theory}

Nonequilibrium steady states of oxidation/reduction reactions in flow tanks are often probed by measuring the electrochemical potential for the ions in solution. The Nernst equation for the electrochemical potential holds only if the forward and reverse rates are equal for each of the individual electron transfer reaccions at the electrode. Measurable deviacions from the Nernst equation are occur in nonequilibrium systems when more than one redox couple is active at the electrode. In this work, we derive the deviations from the Nernst equation for redox systems in nonequilibrium steady states, by use of macroseopic electrode kinetics. The deviations depend on the displacement of the steady state from equilibrium, the exchange current densities for the active redox couples, and the transfer coefficients for the reactions at the electrodes. This study is being submitted to the J. Phys. Chem (406)

\section{Electrochemical potentials in nonequilibrium steady states: calculations for the minimal bramate systems and comparison with experiments}

We have derived deviations from the Nernst equations for the electrochemical potential in a reacting system in a nonequilibrium steady state. The derivation is based on the Butler-Volmer equations, applied to a system in which redox reactions occur in a solution at steady state. Deviations from the Nernst Equation occur when more than one redox pair is active at an inert 
electrode, which is placed in the reacting solution and connected to a reference electrode through an external circuit to a reference electrode. In this work, we apply the general theory to the minimal bromate oscillator, using the Noyes-Field-thompson mechanism for the elementary steps; nine reactive species are included in the mechanism. We determine the deviations from the Nernst potential, ovaluated for the $\mathrm{Ce}{ }^{\mathrm{IV}} / \mathrm{Ce}$ III couple. The calculated doviations depend on the displacement of the steady state from equilibrium, the ratios of exchange current densities of the various redox couples, and the activity coefficients of the corresponding ions. Experimental measurements of the electrochemical potential have been reported for the minimal bromate system in a tank reactor, operated under steady state conditions, and deviations have been found from the Nernst potential (calculated using spectrophotometric measurements of the $\mathrm{Ce}^{\text {iv }}$ ion concentrations). We find qualitative agteement with the experimental measurements for the minimal systems, with uncertainties in the results due primarily to uncertainties in the exchange current densities for redox couples that are active at the electrode. This study will be submitted to the J. Phys. Chem (407)

\section{Publication accredited to DOE:}

368. "Linear free energy relations and reversible stretched exponential kinetics in systems with static or dynamical disorder", J. Phys. Chem. B. 103, 1563-1580 (1999) Marcel O. Vlad, David L. Huber and John Ross.

369. "Nonlinear kinetics and new approaches to complex reaction mechanisms", Ann. Rev. Phys. Chem., 50, 51-78, (1999), John Ross and Marcel O. Vlad.

"370. Spatial correlations near Turing instabilities; criteria for wavenumber selection", J. Phys. Chem. A. 103, 1347-1356 (1999) William Vance and John Ross.

374. "Measurements of phase response in an oscillatory reaction and deduction of components of the adjoint eigenvector", J. Phys. Chem. A., 103, 8252-8256,(1999), Ian Millett, William Vance, John Ross.

375. "Statistical ensemble approach and fuctuation-dissipation relations for multivariable chemical systems far from equilibrium", J. Phys. Chem, A, 104, 3159-3176, (2000), Marcel 0. Vlad and John Ross.

379. "A master equation approach to synchronization in diffusion-coupled nonlinear oscillators", Phys. Rev. E., 62, No.3, 3303-3310 (2000), William Vance and John Ross

380. "Statistical fractal adsorption isotherms, linear energy relations and power-law trappingtime distributions in porous media", Phys. Rev. E. 62, 837-844, (2000), Marcel Ovidiu Vlad, Gianfranco Cerofolini and John Ross

381. "Lévy diffusion in a force field, Huber relaxation kinetics and nonequilibrium thermodynamics. H-theorem for enhanced diffusion with Lévy white noise", Phys. Rev. E., $\underline{62}$, No.2, 1743-1763, (2000), Marcel O. Vlad, John Ross and Friedemann W. Schneider

382. "New Approaches to Complex Reaction Mechanisms", Design Principles for the Immune Systems and Other Distributed Autonomous Systems, Lee Segel and Irun Cohen, eds., Oxford University Press, 261-277, (2001), John Ross and Marcel Vlad, 
384. "On the Deduction of Chemical Reaction Pathways from Measurements of Time Series of Concentrations", Chaos, 11. 1, 108-114, (2001). Michael Samoilov, Adam Arkin, and John Ross.

388. "Effective medium approximation for Taylor transport in systems with static disorder." Journal of Physical Chemistry, B Marcel O. Vlad, Federico Moran, and John Ross. , 105, 11710$11718(2001)$

389. "Bayesian analysis of systems with random chemical composition: Renormalization-group approach to Dirichlet distributions and the statistical theory of dilution." Marcel Ovidiu Vlad, Masa Tsuchiya, Peter Oefner, and John Ross, Phys. Rev. E., 65, 011112 (2001)

394. "Determination of Thermodynamic and Stochastic Potentials in Non-equilibrium System from Macroscopic Measurements" John Ross, Katherine L.C. Hunc, Marcel O. Vlad, J. Phys. Chem. A, 106, 10951-10960, (2002)

399. "Systematic derivation of reaction-diffusion equations with distributed delays, and relations to fractional reaction-diffusion equations and hyperbolic transport equations." Marcel O. Vlad and John Ross. Phys. Rev. E. 66030102 (2002)

406. "Electrochemical potentials in nonequilibrium steady states: General theory" Katherine L. C. Hunt, Paul M. Hunc, Masanori Yamato and John Ross. [This study is being submitted to the J. Phys. Chem]

407. "Blectrochemical potentials in nonequilibrium steady states: Calculations for the minimal bromate system and comparison with experiments." Katherine L. C. Hunt, X. Li, Paul M. Hunt and John Ross. [This will be submitted to the J. Phys. Chem]

\section{Awards:}

Presidentisl Medal of Science, 1999

Peter Debye Award in Physical Chemistry,American Chemical Society 2001

Austria's Cross of Honor for Science \& Art First Class, 2002 